\title{
Magnetic fields of A-type stars
}

\author{
G. Mathys
}

European Southern Observatory, Casilla 19001, Santiago 19, Chile

\begin{abstract}
The current status of our knowledge of magnetic fields in A-type stars is reviewed with special emphasis on the progress achieved for "classical" Ap stars over the last four years. For those stars, the distribution of the strength of the field, its three-dimensional structure, and its relation with rotation and evolution are discussed.
\end{abstract}

Keywords. Stars: magnetic fields, stars: chemically peculiar, stars: rotation, stars: evolution

\section{Introduction}

A-type stars occupy a special place in the history of the study of stellar magnetism. Indeed, it is in such a star, $78 \mathrm{Vir}$, that the first detection of a magnetic field in a star other than the Sun was achieved (Babcock 1947). That Babcock had selected a peculiar star (78 Vir was classified A2p) as his target of choice did not reflect any a priori assumption about the existence of a connection between peculiarity and magnetic field. Instead it relied on the hypothesis that fast rotating stars could generate strong magnetic fields and it was driven by the consideration that the small effects of magnetic fields could be better detected in sharp spectral lines than in broad ones. Babcock originally believed that 78 Vir was a fast rotating star whose rotation axis lies (almost) parallel to the line of sight. To this date (fairly) sharp-lined stars remain privileged targets for magnetic field studies. Among the stars in which magnetic fields have been found, Ap stars still represent the most numerous group. However, the overall picture of stellar magnetism that has progressively emerged since Babcock's ground-breaking discovery corresponds to a physical reality very remote from the reasoning underpinning his detection attempt. $\dagger$ An overview of our current knowledge and understanding of A star magnetic fields, based on the wealth of observational results that have been obtained over the years, is given in this presentation. Important open questions, whose answers require additional observational constraints, are emphasised.

For a long time, Ap and Bp stars were the only non-degenerate stars besides the Sun in which magnetic fields had been definitely detected and measured. Occasionally, detections of magnetic fields in stars of other types in the same temperature range were reported, but in general they could not be confirmed in subsequent, independent studies. The continued development of instrumentation that brought about the breakthrough discovery of solar-like magnetic fields in the late-type dwarfs $\xi$ Boo A and 70 Oph A (Robinson et al. 1980) triggered renewed interest in attempts to detect magnetic fields in non-peculiar A stars, taking advantage of the ever increasing accuracy achievable. Naturally, considering the apparent relation of their chemical peculiarities to those of Ap stars and their predominantly slow rotation, Am stars and HgMn stars were primary targets for such investigations. The first "modern" study of this kind was conducted by Mathys \& Lanz (1990), who concluded to the probable presence of a magnetic field of the

$\dagger$ It is an amazing, and quite fortunate coincidence that the order of magnitude of the Ap star magnetic fields is similar to the one Babcock had come to expect on the basis of an incorrect reasoning. 
order of $2 \mathrm{kG}$ in the hot Am star $o$ Peg, from consideration of the differential broadening of spectral lines with different magnetic sensitivities and of what appeared to be differential magnetic intensification between the two Fe II lines $\lambda \lambda 6147.7$ and 6149.2. Analysis of the same line pair led Lanz \& Mathys (1993) to suggest that magnetic fields are also present in two other hot Am stars, HD 29173 and HD 195479A. More recently, the same method was applied by Hubrig et al. (1999) and by Hubrig \& Castelli (2001) to look for magnetic fields in a sample of HgMn stars and of superficially normal late B stars. The analysis relies on modern spectrum synthesis techniques and on recent determinations of the $g f$ values to show how critically the conclusions depend on the accuracy of the latter. Yet, it suggests that detectable magnetic fields may be present in some of the studied stars. If so, they must have a geometric structure sufficiently complex so as to remain undetected with the unprecedented sensitivity achieved in the recent circular spectropolarimetric observations of Shorlin et al. (2002). The median $1 \sigma$ uncertainty of the mean longitudinal magnetic field $\ddagger$ determinations achieved by these authors are $18 \mathrm{G}$ for Am stars, $39 \mathrm{G}$ for $\mathrm{HgMn}$ stars, and $13 \mathrm{G}$ for normal A and B stars. However, Moninet et al. (2002) report the probable detection of a longitudinal field of $-54 \pm 12 \mathrm{G}$ in the normal F7V star $\chi$ Dra. On the other hand, the recent discovery (Wahlgren et al. 2001, Adelman et al. 2002) of periodic profile changes in the line $\mathrm{Hg}$ II $\lambda 3984$ of the $\mathrm{HgMn}$ star $\alpha$ And, if interpreted as due to inhomogeneities in the distribution of $\mathrm{Hg}$ on the surface of this star (as seems most plausible), requires the existence of a mechanism of horizontal differentiation of the atmospheric composition, which suggests that a magnetic field with sufficient large-scale organisation may be present in this star.

In summary, despite the increase in the achievable sensitivity and accuracy for magnetic field detection and determination that has taken place over the last 15 years, the question of the presence of magnetic fields in non-peculiar A stars, in Am stars, and in HgMn stars remains open. Accordingly in the rest of this review, I shall focus my attention on what is still the only class of A stars where the existence and the properties of magnetic fields are well established and documented: the Ap stars. More specifically, I shall consider explicitly only the "classical" Ap stars, or CP2 stars, in Preston's (1974) naming convention, to the exclusion of the hotter He weak and He strong stars, which as B stars fall outside the scope of this symposium. Yet one should keep in mind the close relation they bear to the Ap stars proper. Most of what refers to the latter also apply to the former. On the other hand, the sequence of the classical Ap stars actually stretches from late B to early $\mathrm{F}$ spectral types, and it would make no sense to exclude its coolest and hottest members from the discussion even though they are not strictly speaking A-type stars.

\section{Ap star magnetic fields: overview}

A review of the status of knowledge and understanding of Ap star magnetic fields as of end of 2000, with some emphasis on the way in which the presented conclusions were reached, can be found in Mathys (2001). I shall only summarise here the main results reported in this paper, referring the reader to it for more details. In this presentation, I emphasise the progress that has been made and the questions that have been debated since then.

Magnetic fields of Ap stars cover the whole stellar surface. They have a significant degree of large-scale organisation, such that the range of field strengths over the stellar surface is relatively narrow. In first approximation, their structure is roughly symmetric

$\ddagger$ The mean longitudinal magnetic field is the line-intensity weighted average over the visible stellar hemisphere of the component of the magnetic field along the line of sight. 
about an axis that is inclined to the stellar rotation axis, Ap stars are oblique rotators. In most cases this structure bears some resemblance to a simple dipole encompassing the whole star. Intrinsic variations of Ap star magnetic fields have not been definitely observed so far. The only variations that are observed are periodic and result from the changing aspect of the visible stellar hemisphere as the star rotates.

The magnetic field moment for which the largest number of measurements has been obtained is the mean longitudinal magnetic field. Its measured values range (in absolute value, since it can be either negative or positive) from less than $100 \mathrm{G}$ up to $20.5 \mathrm{kG}$ in Babcock's star HD 215441 (Borra \& Landstreet 1978). Bychkov et al. (2003) show that the observed distribution of the rms longitudinal field (Borra et al. 1983) of Ap stars is consistent with a decreasing exponential. It is quite plausible, but cannot be fully ascertained at present, that the breakdown of this distribution below $100 \mathrm{G}$ reflects the currently achievable limit of sensitivity of longitudinal field determinations. The latter has become progressively lower over the past years (e.g., Wade et al. 2000, Shorlin et al. 2002), allowing a growing number of weak magnetic fields to be definitely detected and accurately measured. Some of the most accurate longitudinal field measurements obtained to this date suggest that all Ap stars are magnetic and that there may exist a minimum field strength for which Ap-type characteristics are produced (Aurière \& Wade 2005). At the other end of the distribution, while Babcock's star, which has been known for many years, still stands by quite some margin as the record holder, a couple of additional stars showing exceptionally large longitudinal fields have recently been discovered: HD 178892 (B9p $\mathrm{SrCrEu}$ ), with measured values of up to $8.5 \mathrm{kG}$ (El'kin et al. 2003), and NGC 2244-334 (a Bp He weak star), for which a single measurement yielded a longitudinal field of $-9.0 \mathrm{kG}$ (Bagnulo et al. 2004). The longitudinal fields of these two Bp stars are stronger than any other known so far, save Babcock's star. El'kin et al. $(2002,2003)$ found three more stars with longitudinal fields greater than $3 \mathrm{kG}$, and four others where the largest (in absolute value) measurement of the longitudinal field is between 1.5 and $3.0 \mathrm{kG}$. Their contribution considerably increases the number of Ap stars known to have strong longitudinal fields. Another recently found member of this group is HD 66318 (= NGC 2516-24) for which Bagnulo et al. (2003) report a longitudinal field value of $4.5 \mathrm{kG}$. Strongly magnetic Ap stars are interesting because they provide opportunities to study the effect of the magnetic field with the greatest detail and accuracy.

Due to its strong dependence on the geometry of the observation, and its resulting often large variability over a rotation period, the mean longitudinal magnetic field is poorly suited to characterise the actual stellar field. To this effect, a much more appropriate field moment is the mean magnetic field modulus, that is, the line-intensity weighted average over the visible stellar hemisphere of the modulus of the magnetic field. It is determined from measurement of the separation of the $\sigma$ (or $\sigma$ and $\pi$ ) components of lines that show resolved magnetic splitting. Such lines can be observed only in stars that have a sufficiently strong field and that rotate slowly enough so that the magnetic splitting is not smeared out by rotational Doppler effect. This, of course, restricts the number of stars in which the mean field modulus can be determined. At the time of writing, to the best of this author's knowledge, this number is 47. It includes the 42 stars discussed by Mathys et al. (1997), HD 18610 (Stütz et al. 2003), HD 66318 (Bagnulo et al. 2003), HD 51684 and HD 213637 (Mathys et al. , in preparation†), and HD 94427 (unpublished;

$\dagger$ Resolved magnetically split lines were found in HD 213637 by these authors on a spectrum taken on November 11, 1997. This finding was announced by the present author in his review presented at the conference on "Magnetic fields across the Hertzsprung-Russell diagram" held in Santiago in January 2001. Mathys (2003) reports that a total of 7 high-resolution spectra of 
the line Fe II $\lambda 6149.2$ was observed to be resolved in a spectrum taken on February 26, 2000). Mathys (2003) pointed out that 7 of the stars where magnetically resolved lines have been observed belong to the group of the rapidly oscillating Ap (roAp) stars. The most recent addition, HD 116114 (Kurtz et al. 2005), also shows lines resolved by the magnetic field. The roAp stars with resolved magnetically split lines represent more than $20 \%$ of all known roAp stars. This indicates an unusually high frequency of occurrence of strong magnetic fields and of slow rotation among roAp stars, compared to Ap stars in general.

A remarkable result of the extensive study of stars with magnetically resolved lines conducted by Mathys et al. (1997) is the apparent existence of a low-field cutoff around $2.8 \mathrm{kG}$ in the distribution of the mean field modulus, averaged over the rotation period (for more details see the original reference, and Mathys 2001). Four of the five additional stars with resolved magnetically split lines have mean field moduli exceeding $5 \mathrm{kG}$. The only exception is HD 94427, where the field modulus measured in February 2000 is $2.2 \mathrm{kG}$. This single measurement does not by itself question the existence of a low-field cutoff in the field modulus distribution. Indeed it may just correspond to a rotation phase close to the minimum of the field modulus, such that the average of the latter over the rotation period is significantly higher and above the cutoff. This is, as matter of fact, observed in other stars (e.g., HD 59435: see Wade et al. 1999). Mathys et al. (1997) established that the $2.8 \mathrm{kG}$ lower limit of the distribution of the mean field modulus (averaged over the rotation period) is well above the limit of resolution of the diagnostic line Fe II $\lambda 6149.2$. But they could not definitely decide if this lower limit represents a cutoff or a discontinuity in the distribution. They mention the observation of a number of stars in which no magnetic line splitting could be resolved observationally. At least 14 of the latter have $v \sin i$ small enough so that smearing out of magnetically resolved line components by rotational Doppler effect can be ruled out. Of these 14 stars, only two have been observed in circular polarisation to measure their mean longitudinal magnetic field: HD 8441 and HD 176232. Mathys \& Hubrig (1997) did not definitely measure a non-zero longitudinal field in the latter, and the significance of Babcock's (1958) measurements of this field moment depends on the correct estimation of their uncertainties. Suspicion that the latter are underestimated for HD 176232 is strengthened by the consideration that this is almost certainly the case for Babcock's (1958) determinations of the longitudinal field of HD 8441, as they would otherwise be irreconcilable with its $69.5 \mathrm{~d}$ rotation period, which is securely established from analysis of photometric variations (Wolff \& Morrison 1973). On the other hand, Preston (1970) refers to 9 high quality measurements of the longitudinal field of HD 176232 obtained on 10 consecutive nights in 1970, which indicate a constant value of $500 \pm 100 \mathrm{G}$; the lack of further details does not allow a critical evaluation of their significance. In other words, in a couple of cases, circular spectropolarimetry gives some hint of, but does not definitely establish, the presence of weak detectable magnetic fields in sharp-lined Ap stars where no magnetic line splitting or broadening has been observed in high-resolution spectra recorded in unpolarised light. On the other hand, line synthesis suggests the presence of a magnetic field of the order of $1 \mathrm{kG}$ in HD 176232 (Ryabchikova et al. 2000), which is fully consistent with the upper limit resulting from the study of Mathys et al. (1997). A somewhat larger value, $1.5 \mathrm{kG}$, was derived through a similar analysis by Kochukhov et al. (2002). But the most definite proof so far of the presence of a fairly weak magnetic field in HD 176232 is due to the high-resolution infrared observations of Leone et al. (2003) from which a mean

this star had been obtained by end of 2002. An independent report of the discovery of resolved lines, based on a spectrum taken on April 17, 2002, was published by Kochukhov (2003). 
field modulus of $1.4 \mathrm{kG}$ is diagnosed. As a matter of fact, high-resolution spectroscopy in the infrared is the royal way for future studies of the low end of the magnetic field strength distribution. Indeed the quadratic wavelength dependence of the Zeeman effect (opposed to the linear dependence of the Doppler effect) implies that for a given $v \sin i$, resolved magnetically split lines can be observed for lower fields than in the visible, and conversely, that magnetic line resolution is observable at a given field strength for faster rotators in the infrared. With high-resolution infrared spectrographs now coming of age, such as CRIRES at ESO's VLT, a new era opens for the study of stellar magnetism.

\section{Magnetic field structure}

Modelling of the geometric structure of the magnetic fields of Ap stars has long been limited by the crudeness of the observational constraints that could be obtained. Until the advent of modern CCD detectors, magnetic field diagnosis was for most stars restricted to the determination of their mean longitudinal magnetic field. At the achievable accuracy, its variation in a stellar rotation period did not significantly depart from a sinusoid. With the latter as the only constraint, no model more complex than a single dipole at the stellar centre, with its axis inclined to the stellar rotation axis, can be uniquely derived. In the few fortunate cases where magnetic splitting of spectral lines could be resolved and followed throughout a stellar rotation cycle on spectra recorded on photographic plates, the deficiencies of the centred dipole model were revealed. Additional free parameters were required, leading to the introduction of "perturbations" of the centred dipole, such as the superposition of a dipole and a collinear quadrupole at the centre of the star, with their common axis inclined to the stellar rotation axis, or a dipole offset from the centre of the star by a (relatively small) fraction of the stellar radius in the direction of its axis (not aligned with the stellar rotation axis). Again, models of this type are the most complex ones that could be unambiguously constrained with the observational data available at the time. Systematic exploitation of the information contents of line profiles recorded in circular polarisation began in the second half of the 1980s with this author's observations of a sample of magnetic Ap stars with ESO's Cassegrain Echelle Spectrograph (CASPEC) attached to ESO's $3.6 \mathrm{~m}$ telescope (Mathys 1991). Curves of variations along the rotation cycle of moments of the stellar magnetic fields, as derived from measurements of low-order moments of the observed Stokes $I$ and $V$ line profiles (Mathys 1995a, 1995b, Mathys \& Hubrig 1997, Mathys et al. in preparation), possibly combined with curves of variation of the mean magnetic field modulus, for those stars with magnetically resolved lines (Mathys et al. 1997), or with broad band linear polarisation measurements (Leroy 1995), proved amenable to the derivation of models of the field structure of greater complexity, such as the superposition of collinear dipole, quadrupole and octupole components at the star centre (Landstreet \& Mathys 2000) or that of a dipole and a (nonlinear) quadrupole at the star centre (Bagnulo et al. 2000, 2002b). However, the shortcomings of these models soon became evident when the linear polarisation line profiles that they predicted were confronted with newly obtained high resolution, high signal-to-noise, Stokes $Q$ and $U$ observations (Bagnulo et al. 2001). This work provides the most compelling concrete demonstration that observations in all four Stokes parameters are necessary to fully constraint the magnetic field geometry. This had been realised, in principle, for a long time, but the extent to which simple models based only on Stokes $I$ and $V$ spectra fail to represent the actual structure of the magnetic field as revealed by the addition of Stokes $Q$ and $U$ observations had not been previously proven. This underlines the need for observations in all four Stokes parameters. While circular polarisation is in first approximation proportional to the (line-of-sight component) of the magnetic field, linear polarisation is a second-order effect in the field strength (see 
e.g., Mathys 2002). Furthermore, in disk-integrated observations, Stokes $Q$ and $U$ signals from various parts of the visible stellar hemisphere cancel out more effectively than their Stokes $V$ counterparts. Accordingly, considerably higher signal-to-noise is required for significant detection and characterisation of line profiles in linearly polarised light than in circularly polarised light. Only recently have the required observations become feasible. The publication by Wade et al. (2000) of the first systematic spectropolarimetric measurements in all four Stokes parameters of a sizable sample of Ap stars with, for a fraction of them, adequate phase coverage, represents a breakthrough in the study of the magnetism in this type of stars. This takes place at a time when the Magnetic Doppler Imaging (MDI) techniques have reached the stage of maturity (Piskunov \& Kochukhov 2002) and developments in computer technology allow one for the first time to fully exploit them to synthesise simultaneously in all four Stokes parameters high-resolution (e.g., $\lambda / \Delta \lambda \sim 30000$ ) profiles of a representative number of spectral lines recorded at enough phases to sample adequately their variations along the stellar rotation period.

The analysis of 53 Cam by Kochukhov et al. (2004a) represents the state of the art in this area. Besides the simultaneous consideration of all four Stokes parameters, the essential difference between MDI and earlier attempts at deriving models of Ap star magnetic fields is that the former does not make a priori assumption about the field structure (e.g., some superposition of low-order multipoles). Yet, a regularisation function is required to ensure consistency between the number of free parameters and the information contents of the available observational data, and to maintain numerical stability. The choice of this function is based on an overall knowledge of the type of field structure under consideration (large-scale organisation for Ap stars, high fractionation for late-type stars). But the derived model seems independent of the details of this choice, so that the representation of the stellar magnetic field that it yields is unambiguous.

Kochukhov et al. (2004a) find that the structure of the magnetic field of 53 Cam is much more complex than any low-order multipole expansion. They regard this result as questioning the validity of such expansions as used so far. However, the existence of some rough axial symmetry in the vast majority of Ap stars seems inescapable unless one adopts the view that statistical correlations established through use of predominantly dipolar models, such as between the inclination of the rotation axis with respect to the rotation axis and the stellar rotation period (see Sect. 5), are purely coincidental, which is not a very sustainable scientific attitude.

\section{The third dimension}

Modelling attempts as described in the previous section are mostly restricted to mapping the horizontal distribution of the strength and orientation of the magnetic vector over the stellar surface. While the possible existence of observable radial gradients of the magnetic field in Ap star photospheres has been debated since more than a quarter of a century (see, e.g., Wolff 1978), this question has in the past few years taken increased relevance, both because of the progress achieved in the knowledge of the vertical variation of other physical parameters and thanks to the improved observational prospects opened by the developments that have taken place in both the instrumental and theoretical areas. Interpretation of the impossibility to account for the strengths and shapes of observed spectral line profiles by adopting a unique value for the abundance of a given element as the manifestation of vertical abundance stratification (e.g., Bagnulo et al. 2001, Ryabchikova et al. 2002) must be regarded with some caution as long as the (vertical) atmospheric structure is not securely established. Indeed, observations of Core-Wing Anomalies in Balmer lines of cool Ap stars indicate that the latter may depart considerably from standard model atmospheres (Cowley et al. 2001). 
Recently, Nesvacil et al. (2004) tried to determine the mean magnetic field modulus on both sides of the Balmer jump for a small sample of Ap stars with resolved magnetically split lines. This is a similar strategy to that of Wolf (1978), except that the field modulus, rather than the longitudinal field, is considered. The significance of the results is limited by the number of suitable diagnostic lines on the short wavelength side of the Balmer jump, but there is a clear hint of stronger fields (with a difference of few hundred Gauss) below the Balmer discontinuity, suggesting that the field increases from lower to higher atmospheric layers. By contrast, Romanyuk (2005) using Wolf's (1978) original approach (albeit with a CCD rather than photographic plates), finds that the magnetic field of $\alpha^{2} \mathrm{CVn}$ decreases with height. On the other hand, the short vertical wavelength of the pulsation modes of roAp stars (Kurtz et al. 2005 and references therein) allows one to use the pulsation amplitudes and phases derived for individual spectral lines as atmospheric depth proxies. This opens the prospect to investigate the depth dependence of the magnetic field by relating values determined from such lines with their formation depth. While this approach has not been applied yet, controversy has arisen in the last year about the results obtained through a different use of pulsation observations to diagnose the possible existence of vertical magnetic field gradients. Namely, as a result of the pulsation, the line-forming region moves up and down, hence samples different depths in the atmosphere. If the magnetic field strength vertical variation is sufficient over the amplitude of this motion, field values derived from consideration of a given line should vary with the pulsation period. Attempts to detect such variations based on longitudinal field determinations yielded inconsistent results. Hubrig et al. (2004), applying the method introduced by Bagnulo et al. (2002a) to diagnose the field from low-resolution spectropolarimetric observations with FORS1 at the VLT, did not find any variation at a level of the order of $100 \mathrm{G}$ over the pulsation cycle of six roAp stars. This sample includes $\gamma$ Equ, for which Leone \& Kurtz (2003) report the discovery in Nd III lines of longitudinal field variations with amplitudes of $110-240 \mathrm{G}$ and a period of $12.1 \mathrm{~min}$. This discovery was subsequently questioned by Kochukhov et al. (2004), who obtained an upper limit of 40-60 G for variations with the pulsation period in $13 \mathrm{Nd}$ III lines. Bychkov et al. (2005) using Balmer line photopolarimetry, also fail to detect any variation of the longitudinal field of $\gamma$ Equ with its pulsation period. On the other hand, attempts to detect variations of the field modulus of the same star over its pulsation cycle from Fe II lines observations set upper limits of 5-10 G for such variations (Kochukhov et al. 2004b, Savanov et al. 2005). This is hardly constraining, though, since a rough theoretical estimate suggests that the field modulus variation with the pulsation period might have an amplitude of the order of $11 \mathrm{G}$.

\section{Rotation, binarity, and evolution}

An extensive review of the rotation of A and B type chemically peculiar stars has recently been presented elsewhere (Mathys 2004). It also includes some considerations about binarity. Here I shall only summarise the results that appear most relevant in relation to the magnetic fields of Ap stars. Magnetic Ap stars rotate in average significantly slower than their normal counterparts of similar temperature. Most of them have rotation periods between 1 and 10 days. But there also exists a non-negligible population (possibly of the order of $10 \%$ of all Ap stars) of stars with rotation periods longer than 100 days. The longest periods may exceed one century. These extraordinary slow rotators are interesting because they represent the most extreme manifestation of the braking process that distinguishes Ap stars from normal A stars. One can hope to gain insight into this process by identifying other aspects in which they differ from shorter period stars. Some results of this kind have started to emerge. The conclusion reached 
by Mathys et al. (1997) that the mean magnetic field modulus exceeds $7.5 \mathrm{kG}$ in more than half of the Ap stars with resolved magnetically split lines that have a period shorter than 150 days, but is always smaller than $7.5 \mathrm{kG}$ in longer period stars, remains valid even though a considerable number of additional measurements of this field moment have been accumulated since its publication, and new rotation periods have been determined. Also, the (approximate) symmetry axis of the magnetic field appears to be nearly aligned with the rotation axis in the slowest rotators, while in shorter period Ap stars, the angle between the two axes is usually large (Landstreet \& Mathys 2000, Bagnulo et al. 2002b). A possibly related, intriguing result has been presented at this meeting by Bychkov et al. (2005) who found a bimodal distribution of the angle between the magnetic and rotation axes for a large sample of Ap stars, based on magnetic field measurements from the literature.

On the other hand, while the deficiency of short-period binaries among magnetic Ap stars (compared to normal A stars) is well known (Gerbaldi et al. 1985; Leone \& Catanzaro 1999, Carrier et al. 2002), it has more recently been found (Mathys et al. 1997) that this deficiency is even more pronounced among very slowly rotating Ap stars. Of the 12 Ap stars with rotation period longer than 30 days known to be binaries, only one may have an orbital period (not yet unambiguously determined) shorter than 100 days. In other words, the effectiveness of the achievable rotational braking of Ap stars in binaries appears to be somehow related to the orbital period.

The understanding of the origin and evolution of the magnetic fields of Ap stars, as well as of their slow rotation, requires their evolutionary status to be established. This remains a controversial issue, which cannot be fully discussed within the scope of this review. I shall restrict myself to pointing out some recent results. The conclusion drawn by Hubrig et al. (2000), that magnetic Ap stars with masses below $3 M_{\odot}$ have completed at least $30 \%$ of their main sequence lifetime, was based on the consideration of a sample comprising stars with magnetically resolved lines and stars for which quadratic magnetic field determinations were available. This sample accordingly consists of stars that have magnetic fields above average, and rotation velocities below average. This bias is overcome in the recent work of Hubrig et al. (2004), who fully confirm the original result of Hubrig et al. (2000), on the basis of two different samples of magnetic Ap stars: stars with longitudinal field measurements in the literature and stars whose longitudinal fields have been recently determined in a dedicated project from spectropolarimetric observations with FORS1 at the VLT. The discovery by Bagnulo et al. (2003) of a strong magnetic field in HD 66318, a $2.1 M_{\odot}$ member of the open cluster NGC 2516 that has completed only $16 \%$ of its main sequence lifetime does not fundamentally challenge the general result of Hubrig and collaborators, since the latter is statistical and does not rule out the existence of some outliers. Furthermore, one can note in the figures of the papers of Hubrig and collaborators that the isochrone $\log t=8.3$ defines in the HertzsprungRussell diagram the early evolution envelope of the region where magnetic Ap stars with masses below $3 M_{\odot}$ yr are found, and that the age limit that it places on such stars is not significantly different from the age of NGC $2516, \log t=8.2 \pm 0.1 \mathrm{yr}$.

In a search for progenitors of magnetic Ap stars, an attempt by Druin et al. (2005) to detect magnetic fields in Herbig Ae/Be stars has so far remained inconclusive. At the other end of stellar evolution, observations of the magnetic white dwarfs from the Sloan Digital Sky Survey are consistent with the view that the highest field white dwarfs evolved from main-sequence Ap and Bp stars (Schmidt et al. 2003). However it has not been possible so far to trace the evolution of magnetic Ap stars from the end of their main sequence lifetime to the white dwarf sage. On the other hand, growing evidence for the existence of a large population of white dwarfs with low and moderate magnetic 
fields (e.g., Aznar Cuadrado et al. 2004) strongly suggests that the bulk of the magnetic white dwarfs cannot be accounted for as descendants of magnetic Ap stars, hence that they must have another origin.

\section{References}

Adelman, S.J., Gulliver, A.F., Kochukhov, O.P., Ryabchikova, T.A. 2002, ApJ 575, 449

Auriere, M., Wade, G. 2005. These Proceedings, EP12

Aznar Cuadrado, R., Jordan, S., Napiwotzki, R., Schmid, H.M., Solanki, S.K., Mathys, G. 2004, $A \mathscr{G} A$ 423, 1081

Babcock, H.W. 1947, ApJ 105, 105

Babcock, H.W. 1958, ApJS 3, 141

Bagnulo, S., Landolfi, M., Mathys, G., Landi Degl'Innocenti, M. 2000, AËA 358, 929

Bagnulo, S., Szeifert, T., Wade, G.A., Landstreet, J.D., Mathys, G. 2002a, A\&\&A 389, 191

Bagnulo, S., Landi Degl'Innocenti, M., Landolfi, M., Mathys, G. 2002b, A\&\&A 394, 1023

Bagnulo, S., Wade, G.A., Donati, J.-F., Landstreet, J.D., Leone, F., Monin, D.N., Stift, M.J. 2001, A\&BA 369, 889

Bagnulo, S., Landstreet, J.D., Lo Curto, G., Szeifert, T., Wade, G.A. 2003, A $\mathscr{E} A$ 403, 645

Bagnulo, S., Hensberge, H., Landstreet, J.D., Szeifert, T., Wade, G.A. 2004, A\&̈A 416, 1149

Borra, E.F., Landstreet, J.D. 1978, ApJ 222, 226

Borra, E.F., Landstreet, J.D., Thompson, I. 1983, ApJS 53, 151

Bychkov, V.D., Bychkova, L.V., Madej, J. 2003, A\&\&A 407, 631

Bychkov, V.D., Bychkova, L.V., Madej, J. 2005, These Proceedings, EP13

Carrier, F., North, P., Udry, S., Babel, J. 2002, A\&A 394, 151

Cowley, C.R., Hubrig, S., Ryabchikova, T.A., Mathys, G., Piskunov, N., Mittermayer, P. 2001, A\&A 367, 939

Drouin, D., Bagnulo, S., Landstreet, J. W. et al. 2005, These Proceedings, EP1

El'kin, V.G., Kudryavtsev, D.O., Romanyuk, I.I. 2002, Astron. Lett. 28, 169

El'kin, V.G., Kudryavtsev, D.O., Romanyuk, I.I. 2002, Astron. Lett. 29, 400

Gerbaldi, M., Floquet, M., Hauck, B. 1985, A\&A 146, 341

Hubrig, S., Castelli, F. 2001, A\&A 375, 963

Hubrig, S., Castelli, F., Wahlgren, G.M. 1999, A\&A 346, 139

Hubrig, S., North, P., Mathys, G. 2000, ApJ 539, 352

Hubrig, S., North, P., Szeifert, T. 2004, in: Astronomical Polarimetry - Current Status and Future Directions, ASP Conf. Ser. (in press)

Hubrig, S., Kurtz, D.W., Bagnulo, S., Szeifert, T., Schöller, M., Mathys, G., Dziembowski, W.A. 2004, A\&BA 415, 661

Kochukhov, O. 2003, A\&A 404, 669

Kochukhov, O., Ryabchikova, T., Piskunov, N. 2004, A\&A 415, L13

Kochukhov, O., Landstreet, J.D., Ryabchikova, T., Weiss, W.W., Kupka, F. 2002, MNRAS 337, L1

Kochukhov, O., Bagnulo, S., Wade, G.A., Sangalli, L., Piskunov, N., Landstreet, J.D., Petit, P., Sigut, T.A.A. 2004a, $A \& B A 414,613$

Kochukhov, O., Ryabchikova, T., Landstreet, J.D., Weiss, W.W. 2004b, MNRAS 351, L34

Kurtz, D.W., Elkin, V.G., Mathys, G., Riley, J., Cunha, M.S., Shibahashi, H., Kambe, E. 2005, These Proceedings, 343

Landstreet, J.D., Mathys, G. 2000, A\& A 359, 213

Lanz, T., Mathys, G. 1993, $A \mathscr{E} A$ 280, 486

Leone, F., Catanzaro, G. 1999, A\&\& 343, 273

Leone, F., Kurtz, D.W. 2003, A\&SA 407, L67

Leone, F., Vacca, W.D., Stift, M.J. 2003, A\& $A$ 409, 1055

Leroy, J.L. 1995, A\&AS 114, 79

Mathys, G. 1991, $A \& A S$ 89, 121

Mathys, G. 1995, A\&A 293, 733

Mathys, G. 1995, A\&A 293, 746 
Mathys, G. 2001, in: G. Mathys, S.K. Solanki \& D.T. Wickramasinghe (eds.), Magnetic fields across the Hertzsprung-Russell diagram, ASP Conf. Ser. vol. 248, p. 267

Mathys, G. 2002, in: J. Trujillo-Bueno, F. Moreno-Insertis \& F. Sánchez (eds.), Astrophysical spectropolarimetry (Cambridge: University Press), p. 101

Mathys, G. 2003, in: L.A. Balona, H.F. Henrichs \& R. Medupe (eds.), Magnetic fields in $O, B$ and $A$ stars: origin and connection to pulsation, rotation and mass loss, ASP Conf. Ser. vol. 305 , p. 65

Mathys, G. 2004, in: A. Maeder \& P. Eenens (eds.), Stellar rotation, IAU Symp. 215, ASP Conf. Ser. (in press)

Mathys, G., Hubrig, S. 1997, A\&AS 124, 475

Mathys, G., Lanz, T. 1990, A\&A 230, L21

Mathys, G., Hubrig, S., Landstreet, J.D., Lanz, T., Manfroid, J. 1997, A\&AS 123, 353

Monin, D.N., Fabrika, S.N., Valyavin, G.G. 2002, A\&A 396, 131

Nesvacil, N., Hubrig, S., Jehin, E. 2004, A\& $A$ 422, L51

Piskunov, N., Kochukhov, O. 2002, A\&A 381, 736

Preston, G.W. 1970, PASP 82, 878

Preston, G.W. 1974, ARA\&A 12, 257

Robinson, R.D., Worden, S.P., Harvey, J.W. 1980, ApJ 236, L155

Romanyuk, I. I. 2005, These Proceedings, EP4

Ryabchikova, T.A., Savanov, I.S., Hatzes, A.P., Weiss, W.W., Handler, G. 2000, A 8 A 357, 981

Ryabchikova, T.A., Piskunov, N., Kochukhov, O., Tsymbal, V., Mittermayer, P., Weiss, W.W. 2002, A\&A 384, 545

Savanov, I., Hubrig, S., Mathys, G. et al. 2005, This meeting, http://www.ta3.sk/IAUS224/PDF/GP10.pdf

Schmidt, G.D., Harris, H.C., Liebert, J., et al. 2003, ApJ 595, 1101

Shorlin, S.L.S., Wade, G.A., Donati, J.-F., Petit, P., Sigut, T.A.A., Strasser, S. 2002, A\&̋A 392, 637

Stütz, Ch., Ryabchikova, T., Weiss, W.W. 2003, A\&\&A 402, 729

Wade, G.A., Mathys, G., North, P. 1999, A\& $A$ 347, 164

Wade, G.A., Donati, J.-F., Landstreet, J.D., Shorlin, S.L.S. 2000, MNRAS 313, 851

Wahlgren, G.M., Ilyin, I., Kochukhov, O. 2001, AAS Mtg. 199, \#135.04

Wolff, S.C. 1978, PASP 90, 412

Wolff, S.C., Morrison, N.D. 1973, PASP 85, 141

\section{Discussion}

KochukHOv: The roAp star HD 166473 has a rotation period of the order of 10 years and the maximum of its mean magnetic field modulus is about $9 \mathrm{kG}$. Does this star fall in the empty region of the field modulus vs. period diagram?

MATHYs: No, because the result that no fields stronger than $7.5 \mathrm{kG}$ are found for stars with rotation periods longer than $150 \mathrm{~d}$ refers to the average of the field modulus over the rotation period. In the case of HD 166473, this average is of the order of $7 \mathrm{kG}$.

PRESTON: In constructing maps of surface magnetic fields, can observational errors introduce spurious fine structure in the maps?

Piskunov: Doppler Imaging (and Magnetic Doppler Imaging) is based on a model of rotational modulation which implies certain changes of the line profiles as a function of phase. For example, solid body rotation would predict that a surface feature at a given longitude and latitude should produce certain distortions to the line profiles. The amplitude and the Doppler shift of these distortions are well defined functions of rotational phase. Spurious details in the real observations are presumably random and thus ignored by the inversion. This situation is similar to fitting a sine curve to a long but noisy data sequence when the period is known a priori. 